\title{
THE MODELLING OF A CAPACITIVE MICROSENSOR FOR BIOSENSING APPLICATIONS
}

\author{
P. H. Bezuidenhout ${ }^{a, b}$, J. Schoeman ${ }^{a}$ and T. H. Joubert ${ }^{b}$ \\ ${ }^{a}$ Dept. of Electrical, Electronic and Computer Engineering, University of Pretoria, South Africa; \\ ${ }^{b}$ Materials Science and Manufacturing, CSIR, Pretoria, South Africa
}

\begin{abstract}
Microsensing is a leading field in technology due to its wide application potential, not only in bio-engineering, but in other fields as well. Microsensors have potentially low-cost manufacturing processes, while a single device type can have various uses, and this consequently helps with the ever-growing need to provide better health conditions in rural parts of the world. Capacitive biosensors detect a change in permittivity (or dielectric constant) of a biological material, usually within a parallel plate capacitor structure which is often implemented with integrated electrodes of an inert metal such as gold or platinum on a microfluidic substrate typically with high dielectric constant. There exist parasitic capacitance components in these capacitive sensors, which have large influence on the capacitive measurement. Therefore, they should be considered for the development of sensitive and accurate sensing devices. An analytical model of a capacitive sensor device is discussed, which accounts for these parasitic factors. The model is validated with a laboratory device of fixed geometry, consisting of two parallel gold electrodes on an alumina $\left(\mathrm{Al}_{2} \mathrm{O}_{3}\right)$ substrate mounted on a glass microscope slide, and with a windowed cover layer of poly-dimethyl-siloxane (PDMS). The thickness of the gold layer is $1 \mu \mathrm{m}$ and the electrode spacing is $300 \mu \mathrm{m}$. The alumina substrate has a thickness of $200 \mu \mathrm{m}$, and the high relative permittivity of 11.5 is expected to be a significantly contributing factor to the total device capacitance. The $155 \mu \mathrm{m}$ thick PDMS layer is also expected to contribute substantially to the total device capacitance since the relative permittivity for PDMS is 2.7. The wideband impedance analyser evaluation of the laboratory device gives a measurement result of $2 \mathrm{pF}$, which coincides with the model results; while the handheld RLC meter readout of $4 \mathrm{pF}$ at a frequency of $10 \mathrm{kHz}$ is acceptable within the measurement accuracy of the instrument. This validated model will now be used for the geometric design and simulation of efficient capacitive sensors in specific biological detection applications.
\end{abstract}

Keywords: capacitive biosensor, parasitic capacitances, capacitive microsensor, microsensor

\section{INTRODUCTION}

Biosensors have a wide range of applications due to the low cost associated with their manufacturing, making them ideal to monitor water conditions and thus improve health in rural parts of the world. There exists various types of biosensors, of which the capacitive biosensor holds significant promise for future applications. One of the common problems associated with current biosensors is the long time to obtain results, ranging from 24 to 48 hours. ${ }^{1,2}$

A capacitive biosensor measures the capacitance of a material, and often more notably the permittivity of the medium used between the electrodes, referred to as the analyte. This type of biosensor has extremely low detection limits and is mostly used to measure antibodies, antigen and DNA. ${ }^{3}$ There are two types of capacitive sensors, labelled and unlabelled. Labelled capacitive sensors are high in cost and cannot produce real-time results. Unlabelled capacitive sensors can make real-time measurements and have low cost manufacturing. ${ }^{3}$ Further to this, there are two types of measurement configurations used for capacitive sensing. The one uses an electrode solution boundary and measures the electrostatic double layer boundary surface capacitance. ${ }^{3}$ The other is referred to as parallel plate electrodes, which consist out of two electrodes which measure the capacitance created by the difference in dielectric properties of various materials. ${ }^{3}$

The capacitive microsensor considered here is based on the parallel plate configuration. However, there are parasitic capacitances present in the microsensor which has to be taken into consideration. The aim of this analytical model is to mathematically describe the parasitic influences on the microsensor and to predict the outcome for different analytes and

Further author information:

E-mail: tjoubert@csir.co.za

Sensors, MEMS, and Electro-Optical Systems, edited by Monuko du Plessis, Proc. of SPIE Vol. 9257, 92570P @ 2014 SPIE · CCC code: 0277-786X/14/\$18 · doi: 10.1117/12.2066384 


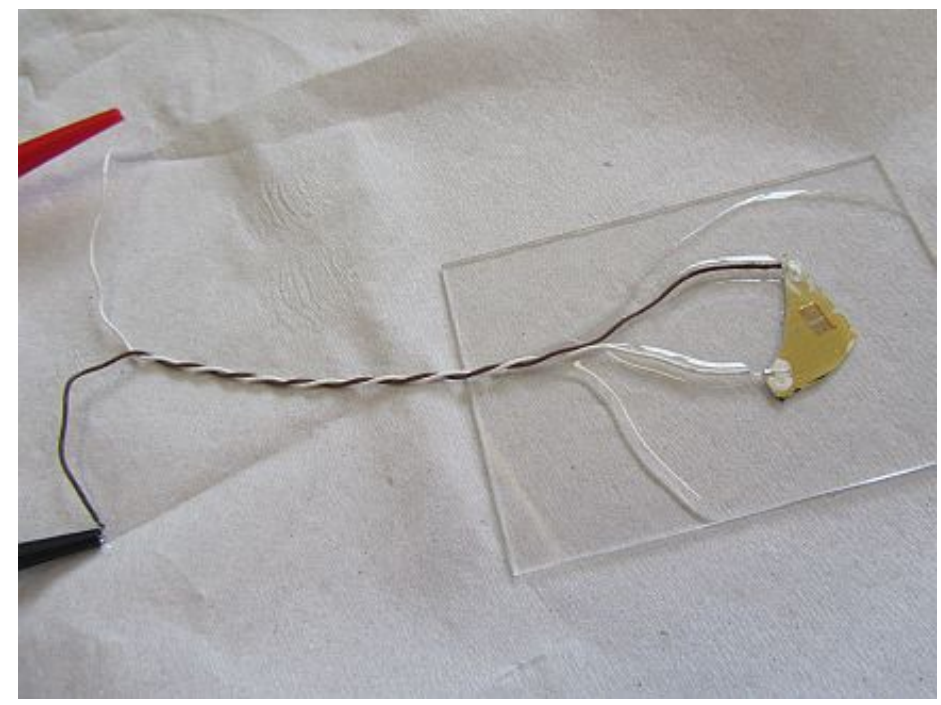

Figure 1: Photograph of the implemented capacitive microsensor.

sensor designs. This enables one to accurately compare a predicted and measured capacitive value for the experimental analyte.

This paper will discuss the development of the analytical model of the microsensor design in Section 2, which will be verified by two experiments as presented in Section 3, and finally concludes with a discussion of the results and findings in Section 4.

\section{MICROSENSOR DESCRIPTION AND MODEL}

\subsection{Microsensor description}

Fig. 1 displays the microsensor that was implemented for this experiment. This microsensor is used to sense the difference in permittivity for possible biosensing applications. The microsensor uses two electrodes, which are implemented in such a way that they act as a parallel plate capacitor. The sensor is mounted on top of a glass microslide.

Fig. 2a illustrates a schematic diagram of the microsensor. The sensor consists out of a three layers mounted on a glass slide. The bottom layer consists of alumina $\left(\mathrm{Al}_{2} \mathrm{O}_{3}\right)$ that provides structural support for the sensor. The second layer consists of a $1 \mu \mathrm{m}$ gold thin film that forms the electrodes after laser cutting a gap. The third layer consists of a PDMS coating, which is used to form the exact dimensions of the receptacle for repeatability. The receptacle is used to measure the permittivity of different analytes, which for this work will only be a baseline measurement of air. The layout of the receptacle is further illustrated in a top view in Fig. 2b. Finally, Fig. 3 shows an equivalent electrical circuit model of the capacitive microsensor. $C_{x}$ is the desired capacitance of the analyte, $C_{s}$ is the constant capacitance due to the parasitic components.

Table 1: The geometric parameters that are used to calculate the parallel plate capacitance of the capacitive microsensor.

\begin{tabular}{|l|c|c|}
\hline Important parameter & \multicolumn{2}{|c|}{ Capacitive microsensor } \\
\hline & Receptacle $\left(w_{0}\right)[\mu \mathrm{m}]$ & Outside receptacle $\left(w_{1}\right)[\mu \mathrm{m}]$ \\
\hline $\mathrm{Au}$ Width $(w)$ & 2643.2 & 6650 \\
\hline $\mathrm{Au}$ Thickness $\left(t_{0}\right)$ & 1 & 1 \\
\hline $\mathrm{Au}$ Diameter $(h)$ & 310.5 & 310.5 \\
\hline $\mathrm{Al}_{2} \mathrm{O}_{3}$ Thickness $\left(t_{\text {sub } 1}\right)$ & 191 & 191 \\
\hline Glass slide thickness $\left(t_{\text {sub } 2}\right)$ & 1000 & 1000 \\
\hline
\end{tabular}




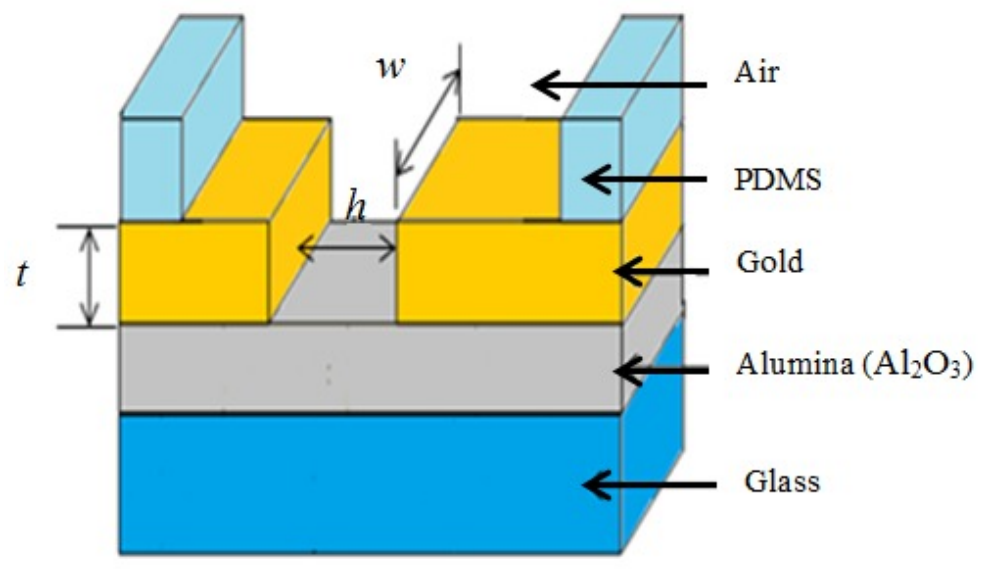

(a) The sectional view of the microsensor.

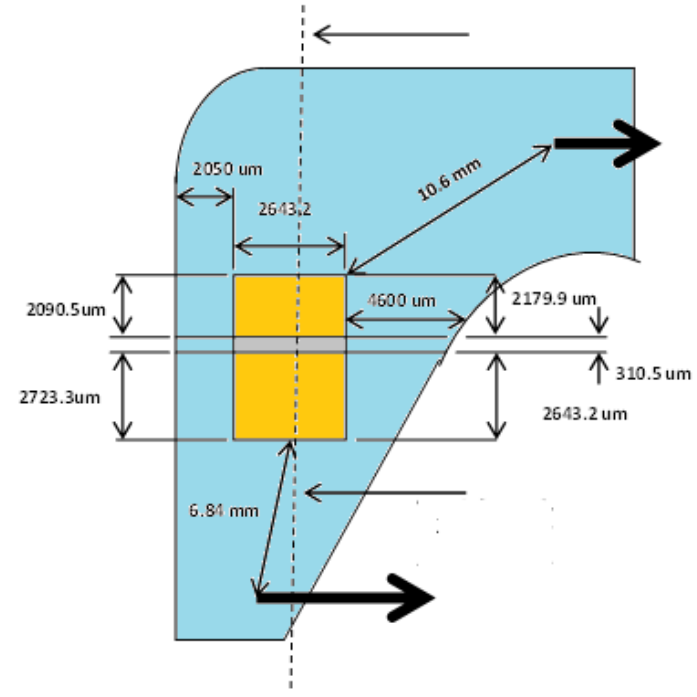

(b) The top view of the microsensor.

Figure 2: The device layout and dimensions.

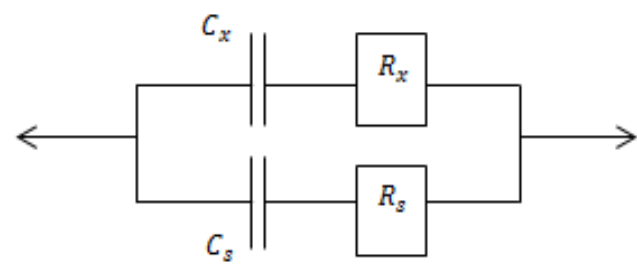

Figure 3: The equivalent electrical circuit of the microsensor.

\subsection{Analytical model derivation for the microsensor}

It has been widely established and accepted that the behaviour of a simple capacitor can be generally described by

$$
C=\frac{\varepsilon A}{d}
$$

where the capacitance is a function of the permittivity, $\varepsilon$, and the device dimensions with $A$ the plate cross sectional area and $d$ the distance between the two parallel plates. However, due to the existence of parasitic capacitances that are caused by additional material layers - necessitated by mechanical support in the capacitive microsensor - a need arises to identify and model the various parasitic components.

Fringing capacitance is created when parallel plate capacitors have finite dimensions, as is always the case for practical systems. The discontinuity in the material results in additional radial components in the electric field, which in turn generate parasitic capacitance components on the sides of a capacitor. This is illustrated in Fig. 4.

Yang $^{7}$ describes an improved capacitance model compared to the classic equation consisting of three terms, the first being a parallel plate capacitor, the second being the fringing field generated by the finite dimensions of the parallel plate capacitor and the third being the fringing field generated by the thickness of the plates. Thus, the overall capacitance as presented by Yang is given by

$$
C=\varepsilon \frac{w}{h}\left(1+\frac{2 h}{\pi w} \ln \left(\frac{\pi w}{h}\right)+\frac{2 h}{\pi w} \ln \left(1+\frac{2 t}{h}+2 \sqrt{\frac{t}{h}+\left(\frac{t}{h}\right)^{2}}\right)\right)
$$


Table 2: The material properties that are used to calculate the parallel plate capacitance of the capacitive microsensor.

\begin{tabular}{|l|c|}
\hline Material & Permittivity \\
\hline Air & $8.85 \times 10^{-12}$ \\
\hline $\mathrm{PDMS}^{4,5}$ & $2.3-2.8 \varepsilon_{0}$ \\
\hline $\mathrm{Al}_{2} \mathrm{O}_{3}{ }^{6}$ & $9.3-11.5 \varepsilon_{0}$ \\
\hline $\mathrm{Glass}^{6}$ & $3.7-10 \varepsilon_{0}$ \\
\hline
\end{tabular}

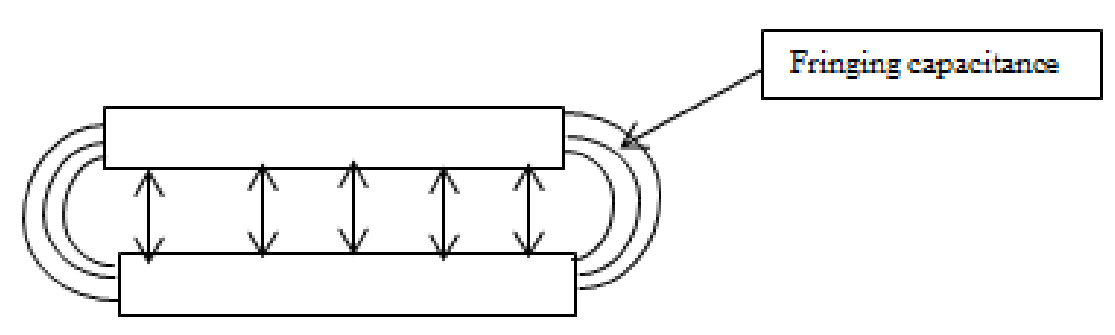

Figure 4: Schematic diagram of the fringing capacitance.

where $C$ is the capacitance, $h$ is the width of the dielectricum, $t$ is the thickness of the plate and $\varepsilon$ is the permittivity of the material inside the receptacle.

However, the above approximation is not accurate when compared to finite element simulations. Leus et al. ${ }^{7}$ investigated the inaccuracy and attribute it to a factor 2 discrepancy in the third term. They propose the use of Palmers equation to replace the first two terms and divide the third term by a factor of two. Palmers model is given by ${ }^{8}$

$$
C=\varepsilon \frac{w}{h}\left(1+\frac{h}{\pi w}+\frac{h}{\pi w} \ln \left(\frac{2 \pi w}{h}\right)\right)
$$

and the final equation proposed for a unit length is given as

$$
C=\varepsilon \frac{w}{h}\left(1+\frac{h}{\pi w}+\frac{h}{\pi w} \ln \left(\frac{2 \pi w}{h}\right)+\frac{h}{\pi w} \ln \left(1+\frac{2 t}{h}+2 \sqrt{\frac{t}{h}+\left(\frac{t}{h}\right)^{2}}\right)\right) .
$$

These formulas are designed for line-to-ground-capacitances. However, Leus et al. show that there exists a zero plane at the symmetrical line of a parallel plate capacitor. ${ }^{7}$ In essence, this means that when considering a unit length for a certain parallel plate gap, $2 h$, the line-to-ground-capacitances and the parallel plate capacitances are related by a factor 2 . This is illustrated in Fig. 5.

The capacitances created by the different substrates, as seen in Fig. 4, occur due to the radial electric field components generated at the electrode borders, which cause the fringing effect in the alumina layer and the glass slide. It is assumed that the electric field, and therefore the capacitance component which extends underneath the glass slide, are insignificant.
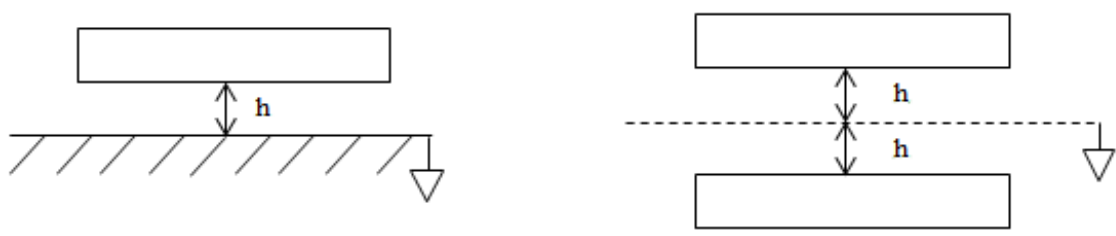

Figure 5: The comparison between the line-to-ground capacitance and the parallel plate capacitance. ${ }^{7}$ 


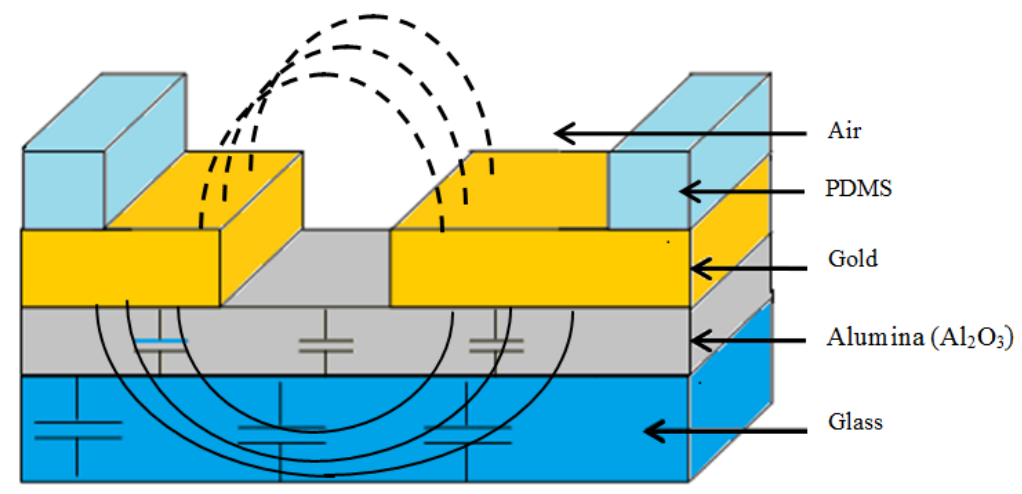

Figure 6: The Schematic 3D section of microsensor (glass slide included) as well as the fringing fields of the microsensor.

In Eq. (4), the permittivity of the glass slide and the alumina layer of the sensor are not taken into account. The desired capacitance is only measured in the receptacle of the sensor. By acknowledging the existence of fringing electric fields in the alumina, the glass and the PDMS layers, the associated capacitances must also be accounted for. This requires that $E q$. (4) is modified for fringing fields that propagate through materials with different permittivities and is illustrated in Fig. 6.

As seen in Fig. 6, the fringing fields propagate through different materials, thus generating different capacitive components. The top half of the fringing field propagates through air (or the measured analyte) and PDMS. Thus, the total fringing component in the calculation of the capacitance is divided into two equal terms, one for the top half and one for the bottom half of the microsensor. At the bottom half of the microsensor, it is assumed that the alumina and the glass divide the bottom fringing component again by approximately two, thus each of these terms is considered four times smaller than the total fringing field. Only the fringing field over the specific width, $w$, as indicated in Fig. 2a of the microsensor is calculated.

When all of the above is considered, the capacitance of the microsensor consists of four linearly contributing unit length capacitive components:

the desired capacitance of the analyte within the receptacle (in this case air)

$$
c_{R}=\varepsilon_{R}\left(\frac{w_{0}}{h}+\frac{\frac{1}{\pi} \ln \left(\frac{2 \pi w_{0}}{h}\right)+\frac{1}{\pi} \ln \left(1+\frac{2 t_{0}}{h}+2 \sqrt{\frac{t_{0}}{h}+\left(\frac{t_{0}}{h}\right)^{2}}\right)+\frac{1}{\pi}}{2}\right),
$$

secondly the parasitic capacitance component contributed by the PDMS coating

$$
c_{a}=\varepsilon_{1}\left(\frac{w_{1}}{h}+\frac{\frac{1}{\pi} \ln \left(\frac{2 \pi w_{1}}{h}\right)+\frac{1}{\pi} \ln \left(1+\frac{2 t_{0}}{h}+2 \sqrt{\frac{t_{0}}{h}+\left(\frac{t_{0}}{h}\right)^{2}}\right)+\frac{1}{\pi}}{2}\right),
$$

thirdly the parasitic capacitance contribution of the alumina substrate

$$
c_{b}=\varepsilon_{2}\left(\frac{w_{t o t}}{h}+\frac{\frac{1}{\pi} \ln \left(\frac{2 \pi w_{t o t}}{h}\right)+\frac{1}{\pi} \ln \left(1+\frac{2 t_{\text {sub1 }}}{h}+2 \sqrt{\frac{t_{s u b 1}}{h}+\left(\frac{t_{s u b 1}}{h}\right)^{2}}\right)+\frac{1}{\pi}}{4}\right)
$$

and finally the parasitic capacitance due to the glass slide 


$$
c_{c}=\varepsilon_{3}\left(\frac{w_{t o t}}{h}+\frac{\frac{1}{\pi} \ln \left(\frac{2 \pi w_{t o t}}{h}\right)+\frac{1}{\pi} \ln \left(1+\frac{2 t_{s u b 2}}{h}+2 \sqrt{\frac{t_{s u b 2}}{h}+\left(\frac{t_{s u b 2}}{h}\right)^{2}}\right)+\frac{1}{\pi}}{4}\right) .
$$

It is noteworthy for the above set of equations to recall that the permittivity is material dependent and given by

$$
\varepsilon=\varepsilon_{r} \times \varepsilon_{0}
$$

and that the dimensions are related through

$$
\begin{aligned}
& w_{t o t}=w_{o}+w_{1}, \\
& t_{t o t}=t_{0}+t_{s u b 1} .
\end{aligned}
$$

Finally, it is assumed that all the capacitors are in parallel, therefore

$$
c_{t o t}=c_{a}+c_{b}+c_{c}+c_{R} .
$$

However, the capacitance over the actual dimensions of the microsensor is required, thus the unit length alone will not suffice. The total unit capacitance ( $\left.c_{t o t}\right)$ of the microsensor must be multiplied with the thickness, $t_{t o t}$, as calculated in Eq. (11). Note that $t_{\text {tot }}$ does not include the thickness of the glass slide. Therefore, the capacitance of the microsensor is given by

$$
C_{\mu \text { Sens }}=c_{t o t} \times t_{t o t} .
$$

The expected predicted capacitance graph for the model is linear, since the only variable in the formula is the permittivity of the measured analyte. However the gradient of the linear curve is a function of the dimensions of the microsensor and can therefore be modified to suit various requirements with different design parameters. This is an indication that different dimensions can be selected when designing a microsensor to adhere to specific capacitative permittivity relationship requirements. The expected result is indicated in Fig. 7.

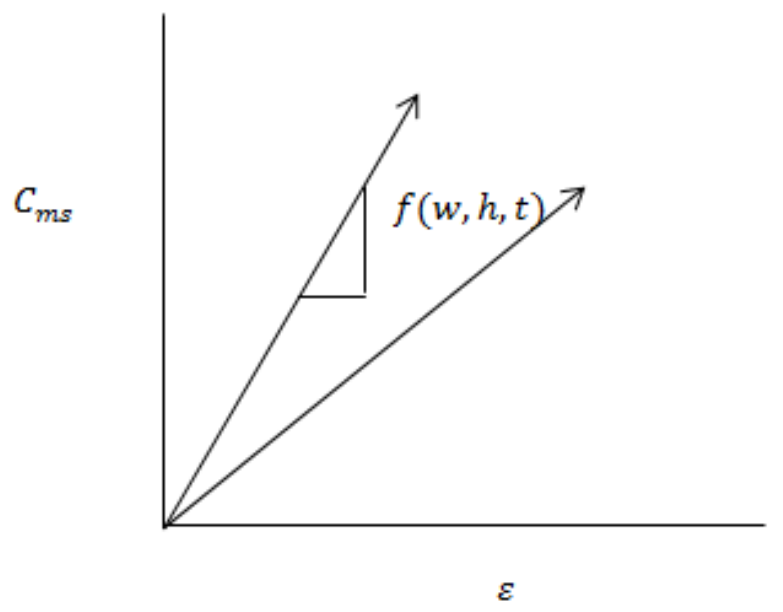

Figure 7: Expected transfer graph for the prediction capacitance to analyte permittivity. 


\section{RESULTS}

Different analytes will exhibit unique material permittivity values. To this end, unique dielectric values are used as input to the capacitive model to calculate the predicted outcome. The values tabulated in Table 1 are used to represent the sensor dimensions. The dielectric constants for PDMS, $\mathrm{Al}_{2} \mathrm{O}_{3}$ and glass were selected from Table 2 as 2.7, 11.5 and 6.7 respectively for the calculation of the parasitic capacitive components.

Table 3: Predicted capacitive output of the model for various permittivities.

\begin{tabular}{|c|c|c|c|}
\hline$\varepsilon_{R}$ & $\varepsilon$ & Example analyte & Capacitance \\
\hline 1 & $8.85 \times 10^{-11}$ & Air & $2.04 \times 10^{-12}$ \\
\hline 2 & $1.79 \times 10^{-11}$ & & $4.20 \times 10^{-12}$ \\
\hline 3 & $2.66 \times 10^{-11}$ & & $6.30 \times 10^{-12}$ \\
\hline 4 & $3.54 \times 10^{-11}$ & E. coli & $8.40 \times 10^{-12}$ \\
\hline 5 & $4.43 \times 10^{-11}$ & & $1.05 \times 10^{-11}$ \\
\hline 6 & $5.31 \times 10^{-11}$ & & $1.26 \times 10^{-11}$ \\
\hline 7 & $6.20 \times 10^{-11}$ & & $1.47 \times 10^{-11}$ \\
\hline 8 & $7.08 \times 10^{-11}$ & & $1.68 \times 10^{-11}$ \\
\hline 9 & $7.97 \times 10^{-11}$ & & $1.89 \times 10^{-11}$ \\
\hline 10 & $8.85 \times 10^{-11}$ & & $2.10 \times 10^{-11}$ \\
\hline
\end{tabular}

The simulated results of the model is plotted in Fig. 8 and show the expected linear relationship between capacitance and permittivity. The gradient of the graph is a function of the dimensions of the microsensor. However, in order to validate the accuracy of the theoretical model, two different experimental methods are performed. The first uses an RLC meter and the second uses an impedance analyser.

\subsection{RLC meter}

The first experiment is done with an RLC meter. However, the theoretically measured capacitive value is close to the RLC meters rails which will cause inaccurate measurements. The RLC meter can also only measure at $1 \mathrm{kHz}, 10 \mathrm{kHz}, 100 \mathrm{kHz}$, $120 \mathrm{kHz}$, while the optimal frequency range of the capacitive microsensor is between $1 \mathrm{MHz}$ and $5 \mathrm{MHz}$. The results are tabulated below.

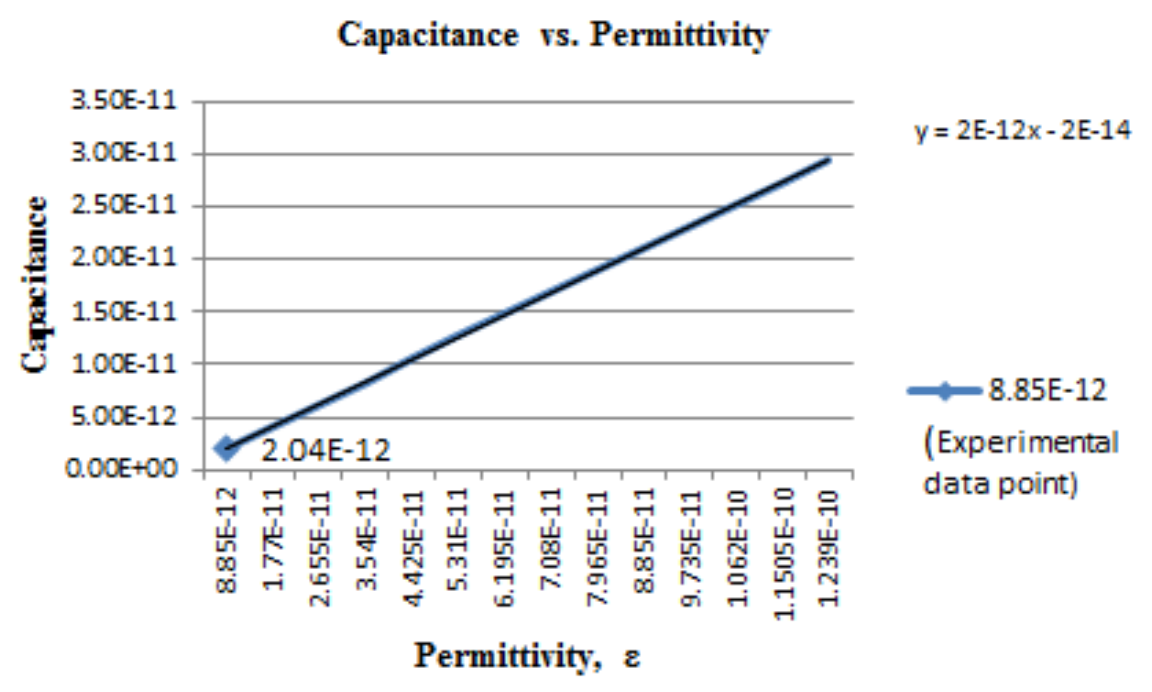

Figure 8: Modelled results of measured capacitance with various input permittivities. 


\section{Capacitance [F]}

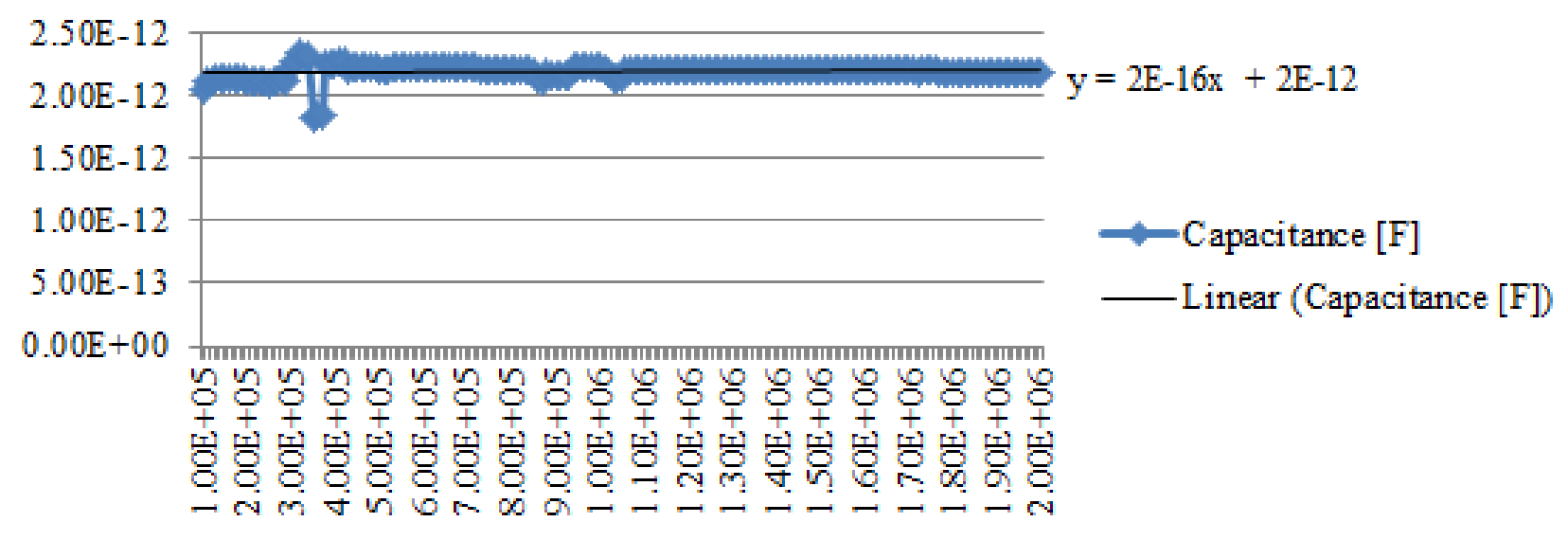

Figure 9: Result obtained from the impedance analyser.

\subsection{Impedance analyser}

The impedance analyser can measure capacitance in the desired frequency range of $1 \mathrm{MHz}$ and $5 \mathrm{MHz}$ and provides sufficient resolution to measure capacitive values into the femto Farad range. As shown in Fig. 9, the magnitude of the capacitance is measured at an almost constant capacitance of $2 \mathrm{pF}$.

The predicted capacitance result $(2.04 \mathrm{pF})$ of the model compares relatively well with the experimental data. In the case where an analyte is selected with a permittivity larger than that of air, it would be possible to directly validate the microsensor capacitance with the RLC meter. However, as shown by the experimental data for the RLC meter, the measurement deviates rather significantly $(3 \mathrm{pF})$ from the predicted model output, motivating the use of the impedance analyser under these circumstances. It should be clear that the impedance analyser provides a sufficiently accurate result ( $2 \mathrm{pF})$ for most practical operating conditions of the microsensor, with the model introducing an error less than $2 \%$. This error can be attributed to the approximations made by the model.

The capacitance calculated using Eq. (1) is in the femto Farad range. This is challenging to measure as the parasitic capacitances are a few orders of magnitude larger than the desired capacitance and thus have a big influence on the measurement. However, by first calculating the capacitive characteristics of air, it can be used as a reference measurement that can be utilised for measuring alternate analyte capacitances. The linearity of the capacitance to permittivity graph indicates that the difference of the measured capacitance and the reference capacitance can be used to determine the capacitance of the desired analyte.

\section{CONCLUSION}

The contribution made in this paper is the development of an analytic model which mathematically defines the parasitic fringing capacitance which exists in materials with different permittivities. These parasitic capacitances can be applied to a microcavity structure that can be utilised as a microsensor for biological measurement applications. By effectively modelling these parasitic capacitances, it aids in the understanding and further development and design of improved capacitive biosensors and ultimately sensing amplifiers. Further to this, by altering the dimensions of the receptacle, the relationship of the capacitance to permittivity can be altered as needed and developed with the analytical model discussed. This particular capacitive microsensor is designed for analytes such as E. coli and other similar biomaterials, but as can be seen in the linearity of the models graph, it can also be employed for other applications where knowledge of capacitance or permittivity is required. 


\section{REFERENCES}

[1] A. K. Dwivedi, G. Pendharkar, R. B. Deshmukh, and R. M. Patrikar, "Detection of E. coli cell using capacitance modulation," in COMSOL Conference 2010.

[2] E. B. Jacob, Y. Aharonov, and Y. Shapira, "Bacteria harnessing complexity," Biofilms, vol. 1, pp. 239-263, 102004.

[3] J. P. Davis, "Parasitic capacitance models," 2002.

[4] Polymer Data Handbook. Oxford University Press, 2009.

[5] R. D. Rivers, "Design, fabrication and characterization of an electrostatically actuated mircofluidic valve," Master's thesis, The Faculty of California Polytechnic State University, 2010.

[6] "Dielectric constants of common materials." Online. www.rafoeg. de/20, Dokumentenarchiv/20, Daten/ dielectric_chart.pdf.

[7] V. Leus and D. Elata, "Fringing field effect in electrostatic actuators," tech. rep., TechnionIsrael Institute of Technology, 2004.

[8] W.-C. Chuang, C.-W. Wang, W.-C. Chu, P.-Z. Chang, and Y.-C. Hu, "The fringe capacitance formula of microstructures," Journal of Micromechanics and Microengineering, vol. 22, no. 2, p. 025015, 2012. 